
Cite this: Energy Environ. Sci., 2013, 6,
2112

Received 22nd December 2012

Accepted 10th April 2013

DOI: $10.1039 / c 3 e e 24461 j$

\section{Ultrathin amorphous zinc-tin-oxide buffer layer for enhancing heterojunction interface quality in metal-oxide solar cells $\dagger$}

\author{
Yun Seog Lee, $+^{\mathrm{a}}$ Jaeyeong Heo, $t^{\mathrm{bc}}$ Sin Cheng Siah, ${ }^{a}$ Jonathan P. Mailoa, ${ }^{a}$ \\ Riley E. Brandt, ${ }^{\text {a }}$ Sang Bok Kim, ${ }^{b}$ Roy G. Gordon ${ }^{b}$ and Tonio Buonassisi*a
}

www.rsc.org/ees

\begin{abstract}
We demonstrate a tunable electron-blocking layer to enhance the performance of an Earth-abundant metal-oxide solar-cell material. A $5 \mathrm{~nm}$ thick amorphous ternary metal-oxide buffer layer reduces interface recombination, resulting in sizable open-circuit voltage and efficiency enhancements. This work emphasizes the importance of interface engineering in improving the performance of Earth-abundant solar cells.
\end{abstract}

Thin film solar cells comprising Earth-abundant, non-toxic, and air-stable materials represent a promising class of photovoltaic (PV) devices compatible with terawatts-scale deployment. ${ }^{1-3}$ Cuprous oxide $\left(\mathrm{Cu}_{2} \mathrm{O}\right)$ is one of several candidate materials under consideration with the potential to reach $20 \%$ power conversion efficiency. ${ }^{4,5}$ Doping this material to make it n-type has proven to be challenging, thus a common PV device architecture comprises a $\mathrm{Cu}_{2} \mathrm{O}-\mathrm{ZnO}$ heterojunction structure. However, device efficiencies remain low, with wafer-based $\mathrm{Cu}_{2} \mathrm{O}$ devices (by thermal oxidation of $\mathrm{Cu}$ sheets at $\sim 1010{ }^{\circ} \mathrm{C}$ ) reaching $4.1 \%$ (ref. 6) and thin film $\mathrm{Cu}_{2} \mathrm{O}$ devices (by electrochemical deposition) reaching $1.3 \%$ (ref. 7 ). The open-circuit voltage $\left(V_{\mathrm{OC}}\right)$ of these devices is significantly below the theoretical limit of $\mathrm{Cu}_{2} \mathrm{O}$, due to a low built-in potential caused by non-ideal band alignment between the absorber and transparent conducting oxide (TCO), and a high recombination-current driven by interface-traps. ${ }^{8,9}$

To mitigate the latter voltage-loss mechanism, we introduce a thin $(\sim 5 \mathrm{~nm})$ buffer layer between the absorber and the TCO. This layer serves as an electron-blocking layer, reducing the

\footnotetext{
${ }^{a}$ Massachusetts Institute of Technology, Cambridge, MA 02139, USA. E-mail: buonassisi@mit.edu

${ }^{b}$ Department of Chemistry and Chemical Biology, Harvard University, Cambridge, MA 02138 , USA

${ }^{\circ}$ Department of Materials Science and Engineering, Chonnam National University, Gwangju 500-757, Korea

$\dagger$ Electronic supplementary information (ESI) available. See DOI: $10.1039 / \mathrm{c} 3$ ee $24461 \mathrm{j}$

\$ Both authors contributed equally to this work.
}

\section{Broader context}

Thin film solar cells comprising Earth-abundant elements are promising candidates for renewable energy applications due to their sustainable rawmaterial usage and potential for cost-effective manufacturing. Earthabundant metal-oxide semiconductors comprise an attractive set of materials for photovoltaic applications, given their auspicious optoelectronic properties and chemical stability suitable for transparent conductor, light-absorber, and transparent buffer layers. For instance, cuprous oxide $\left(\mathrm{Cu}_{2} \mathrm{O}\right)$ and zinc oxide $(\mathrm{ZnO})$ form a type-II heterojunction, yet thin film solar cells of this composition often exhibit substantial power conversion efficiency losses deriving from low open-circuit voltage and fillfactor, believed to be caused by junction non-idealities. In this study, a conformal nanometer-scale amorphous zinc tin oxide buffer layer is introduced between the $\mathrm{Cu}_{2} \mathrm{O}$ absorber and the aluminum-doped $\mathrm{ZnO}$ transparent conductor, reducing dark saturation current density by over an order of magnitude, and improving the open-circuit voltage to $0.553 \mathrm{~V}$ and power conversion efficiency to $2.65 \%$. Atomic layer deposition enables precise tunability of buffer layer stoichiometry and conformity to textured surfaces. This amorphous electron-blocking layer approach may be extended to improve the interface quality in other Earth-abundant thin film materials.

magnitude of the recombination current at the absorber-TCO interface. This approach is reminiscent of the Si-based heterojunction with intrinsic thin layer (HIT) devices, which exhibit $V_{\text {OC }}$ superior to the best Si homojunction devices by creating a small energy barrier with a low density of interface-traps. ${ }^{10}$ This energy barrier is expressed as a conduction-band offset $\left(\Delta E_{\mathrm{CB}}\right)$ of the buffer layer relative to the absorber layer; $\Delta E_{\mathrm{CB}}$ must be carefully "tuned" to avoid current losses (stemming from too high $\Delta E_{\mathrm{CB}}$ ) or voltage losses (stemming from a negative $\Delta E_{\mathrm{CB}}$ ). ${ }^{\mathbf{1 1}}$ This tunability can be achieved by using ternary compounds for the buffer layer, whereby the ratio of two cations (anions) typically modifies the conduction (valence) band position. ${ }^{\mathbf{1 2 , 1 3}}$ Judicious composition selection of this ternary compound allows one to simultaneously limit the concentrations of carriers at the interface as well as reduce the interface-trap density, which reduces dark saturation current density $\left(J_{0}\right)$ and increases $V_{\text {OC }}$. To achieve maximum benefit, this layer needs to 
be thin enough to avoid significant optical absorption (current loss) or resistive loss.

In this study, we introduce an ultrathin amorphous zinc tin oxide (a-ZTO) film as an electron-blocking layer to inhibit recombination at the interface between the $\mathrm{Cu}_{2} \mathrm{O}$ absorber and the Al-doped ZnO (AZO) TCO. Amorphous metal oxides are a new class of semiconductors recently highlighted in transparent electronics due to their superior electronic transport properties with high optical transparency. ${ }^{14,15}$ Specifically, aZTO is a non-toxic and scalable material (high elemental abundance), and has shown a field-effect mobility of up to 13 $\mathrm{cm}^{2} \mathrm{~V}^{-1} \mathrm{~s}^{-1}$ with good thermal stability. ${ }^{16}$ Recently, ZTO has shown potential as a Cd-free buffer layer for CIGS solar cells exhibiting performance comparable to devices with CdS buffer layers. ${ }^{17}$ By controlling the atomic composition of the a-ZTO films, we show that the optical and electrical properties of the buffer layer can be tuned and the heterojunction interface quality can be improved. We demonstrate a power conversion efficiency of our thin film $\mathrm{Cu}_{2} \mathrm{O}$ devices of $2.65 \%$ (total area) with an optimized buffer layer, which to our knowledge is the highest efficiency reported so far for solid-state thin film solar cells with electrochemically deposited $\mathrm{Cu}_{2} \mathrm{O}$.

We fabricate $\mathrm{Cu}_{2} \mathrm{O}$-based thin film solar cells in the substrate configuration with ultrathin a-ZTO (or ZnO) buffer layers. Fig. 1 shows the device architecture and corresponding electron microscopy images of these devices. $2.5 \mu \mathrm{m} \mathrm{Cu}_{2} \mathrm{O}$ thin films are deposited by an electrochemical method on patterned Au electrodes. Grains in the $\mathrm{Cu}_{2} \mathrm{O}$ films have (111) preferred orientation when deposited by lactate solution, which results in a highly textured top surface morphology (Fig. 1b) exhibiting reduced optical reflection desirable for photovoltaic applications. ${ }^{18} \mathrm{~A} 5 \mathrm{~nm}$ thick a-ZTO buffer layer and an $80 \mathrm{~nm}$ thick AZO layer are sequentially deposited by ALD at $120{ }^{\circ} \mathrm{C}$. Crosssectional images of the solar cells taken with high-resolution transmission electron microscopy (HR-TEM) show the individual layers clearly (Fig. 1d). The amorphous nature of the ZTO buffer layer is observed in contrast to crystalline $\mathrm{Cu}_{2} \mathrm{O}$ and $\mathrm{AZO}$ layers. It is confirmed that the ALD process enables a highly conformal coverage of a-ZTO and AZO films on the textured $\mathrm{Cu}_{2} \mathrm{O}$ surfaces without pin-holes (Fig. 1c and d). The AZO films exhibit an electrical resistivity of $5.9 \times 10^{-3} \Omega \mathrm{cm}$. Al grids that shadow $\sim 4 \%$ of the device surface area are deposited for topside electrodes. As a control, a baseline device containing an undoped ZnO (instead of a-ZTO) buffer layer grown by ALD at $120{ }^{\circ} \mathrm{C}$ with the same thickness $(5 \mathrm{~nm})$ is also fabricated.

A cyclic amide of tin ((1,3-bis(1,1-dimethylethyl)-4,5dimethyl-(4R,5R)-1,3,2-diazastannolidin-2-ylidene)Sn(II)) as the Sn precursor and diethyl zinc as the Zn precursor enables the low-temperature ALD of a-ZTO thin films. ${ }^{16,19}$ The atomic composition of the a-ZTO buffer layer is varied by choosing the ratio of ALD sub-cycles for $\mathrm{ZnO}$ and $\mathrm{SnO}_{2}$ deposition to be $3 / 1$, $1 / 1$ or $1 / 3$. Atomic concentrations of $\mathrm{Zn}, \mathrm{Sn}$, and $\mathrm{O}$ in the films

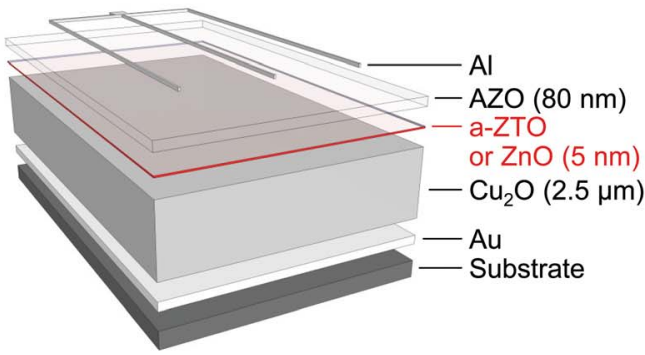

C

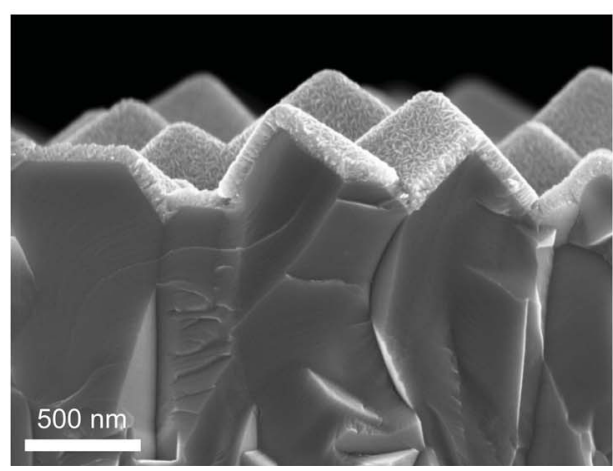

b
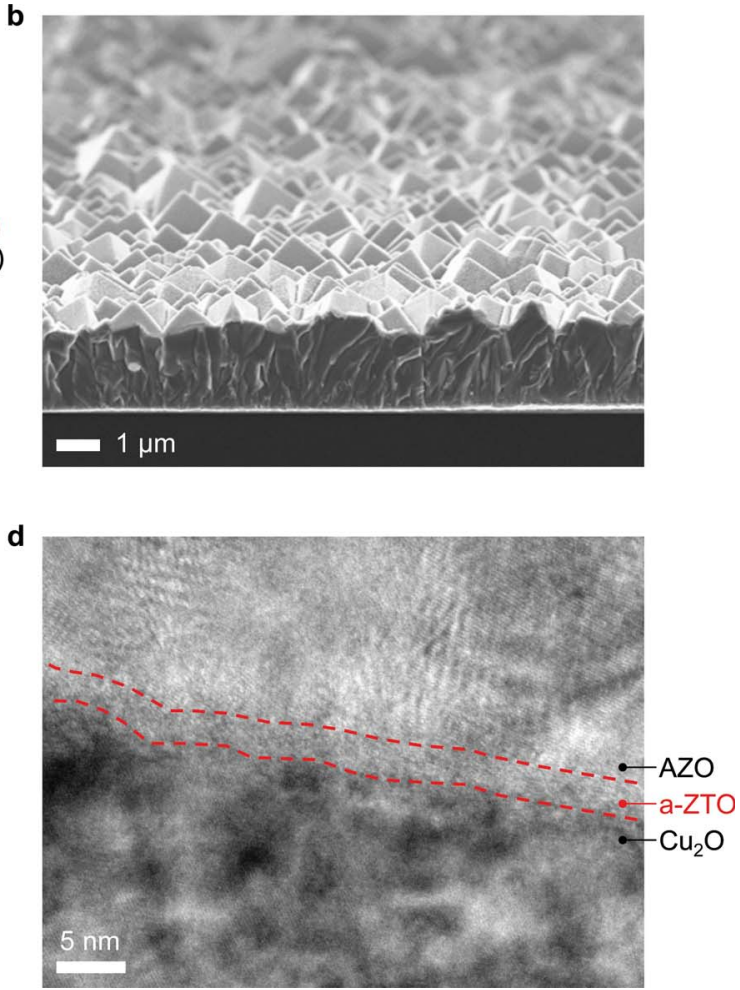

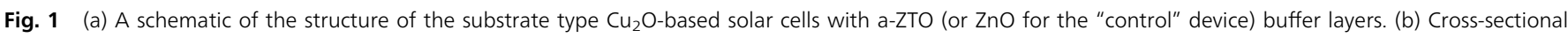

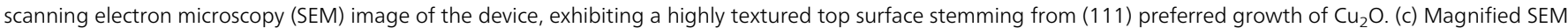

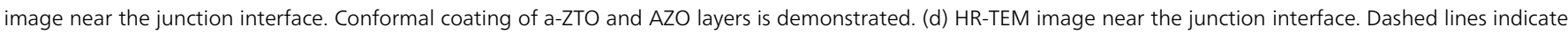
phase boundaries between the amorphous ZTO layer $(\mathrm{Zn} / \mathrm{Sn}=1 / 0.27)$ and surrounding crystalline $\mathrm{Cu}_{2} \mathrm{O}$ and $\mathrm{AZO}$ layers. 
a

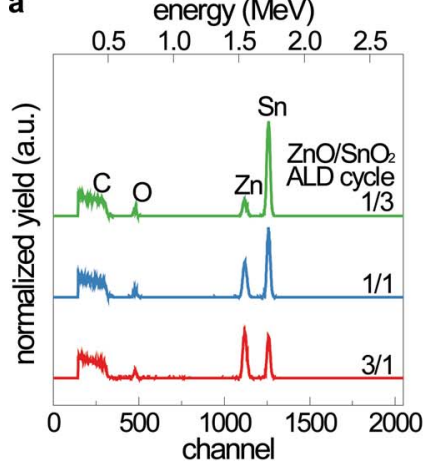

b

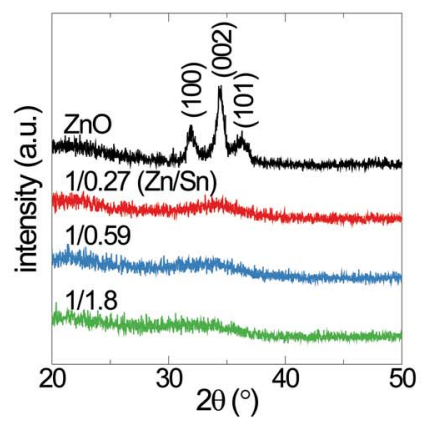

c

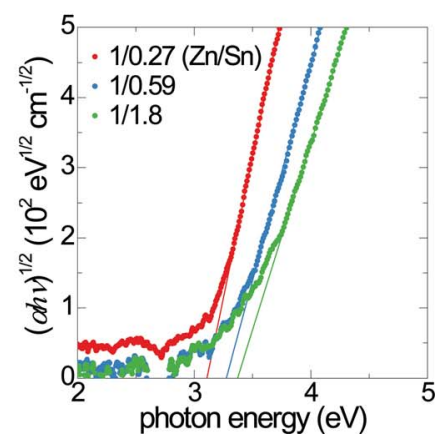

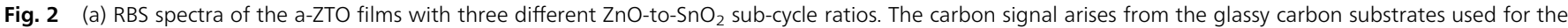

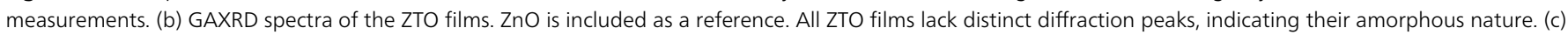
Bandgap extraction from the plot of $(\alpha h \nu)^{1 / 2}$ as a function of photon energy.

are measured by Rutherford backscattering spectroscopy (RBS). Fig. 2a shows the RBS spectra for three different a-ZTO films with different $\mathrm{ZnO}$-to- $\mathrm{SnO}_{2}$ sub-cycle ratios, grown on glassy carbon substrates pre-treated by UV-ozone. No obvious peaks except for $\mathrm{Zn}, \mathrm{Sn}$, and $\mathrm{O}$ from the films and $\mathrm{C}$ from the substrate are observed. The $\mathrm{Zn}$-to-Sn ratio is measured to be $1 / 0.27$, $1 / 0.59$, and $1 / 1.8$ for the films with $\mathrm{ZnO}$-to-SnO ${ }_{2}$ sub-cycle ratios of $3 / 1,1 / 1$, and $1 / 3$, respectively. Oxygen concentrations are 13-19\% higher than the values calculated assuming stoichiometric $\mathrm{ZnO}$ and $\mathrm{SnO}_{2}$. A similar oxygen-rich composition is observed for pure $\mathrm{SnO}_{2}$ ALD using hydrogen peroxide $\left(\mathrm{H}_{2} \mathrm{O}_{2}\right)$ as the oxidant. ${ }^{19}$ The microstructure of the buffer layers is evaluated by glancing-angle X-ray diffraction (GAXRD) as shown in Fig. 2b. Diffraction peaks from the undoped $\mathrm{ZnO}$ film indicate crystalline hexagonal ZnO. On the other hand, all ZTO films are amorphous consistent with HR-TEM measurements. Broad peaks at around $34^{\circ}$ are observed for the three ZTO films, which is characteristic of a-ZTO. ${ }^{\mathbf{1 6 , 2 0}}$ The amorphous nature of these films is a likely consequence of the different cation valences and crystal structures of each pure binary metal oxide; the crystal structures of $\mathrm{ZnO}$ and $\mathrm{SnO}_{2}$ are wurtzite and rutile, respectively. ${ }^{21,22}$

Optical and electrical properties of the a-ZTO films are tuned by varying the Zn-to-Sn ratio. All films exhibit high transmittance in the visible wavelength range and higher transmittance than undoped $\mathrm{ZnO}$ in the UV range (Fig. $\mathrm{S} 1 \dagger$ ). The Tauc model is typically used to determine fundamental bandgap values for amorphous semiconductors. ${ }^{23,24}$ The bandgaps of a-ZTO films are determined from the plot of $(\alpha h \nu)^{1 / 2}$ as a function of photon energy shown in Fig. 2c. As the Zn-to-Sn ratio of the film decreases from $1 / 0.27$ to $1 / 1.8$, the bandgap gradually increases from 3.12 to $3.37 \mathrm{eV}$. Hall measurements reveal an n-type conductivity with an electron density of $3.5 \times 10^{16} \mathrm{~cm}^{-3}$ and a mobility of $3.7 \mathrm{~cm}^{2} \mathrm{~V}^{-1} \mathrm{~s}^{-1}$ for the a-ZTO film with a Zn-to-Sn ratio of $1 / 0.27$, while the other aZTO films with higher Sn contents exhibit resistivities larger than $2 \times 10^{3} \Omega \mathrm{cm}$ and Hall voltages too small to measure, due to a decrease of carrier concentration. ${ }^{16}$ Low carrier concentrations of these films may be due to the strong oxidant, $\mathrm{H}_{2} \mathrm{O}_{2}$, reducing the oxygen vacancy concentration. Similar behaviour

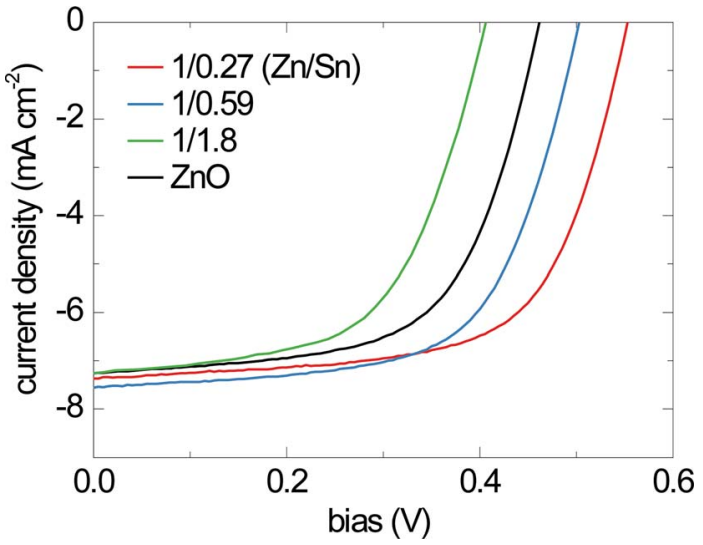

Fig. 3 Current-voltage characteristics of the devices with different a-ZTO buffer layer cation ratios under 1 Sun illumination (AM1.5G spectrum).

is also reported in sputtered a-ZTO layers. ${ }^{25,26}$ Given the low carrier concentration, our simulations suggest that a $5 \mathrm{~nm}$ thick buffer layer does not contain a sufficient amount of charge to change the built-in potential of the device. The undoped $\mathrm{ZnO}$ as a control buffer layer exhibits a resistivity of $2.0 \times 10^{-2} \Omega \mathrm{cm}$, with an electron density of $1.8 \times 10^{19} \mathrm{~cm}^{-3}$ and a mobility of $17 \mathrm{~cm}^{2} \mathrm{~V}^{-1} \mathrm{~s}^{-1}$.

Current-voltage characteristics of the solar cells with a-ZTO and $\mathrm{ZnO}$ (control) buffer layers are acquired under AM1.5G illumination (100 $\mathrm{mW} \mathrm{cm}^{-2}$ ), as shown in Fig. 3. Characteristic device performance properties for each buffer layer composition are summarized in Table 1 . These devices show stable performance upon exposure to air. A strong dependence of $V_{\mathrm{OC}}$ on the incorporated buffer layer material is observed, while shortcircuit current densities $\left(J_{\mathrm{SC}}\right)$ remain nearly constant (7.2-7.5 $\mathrm{mA} \mathrm{cm}{ }^{-2}$ ). The $J_{\mathrm{SC}}$ matches the integrated value of measured external quantum efficiency (EQE) data with the AM1.5G spectrum. A device with an a-ZTO buffer layer comprising a Zn-to-Sn ratio of $1 / 0.27$ exhibits the highest $V_{\text {OC }}$ of $0.553 \mathrm{~V}$, with a fillfactor of $65.0 \%$, and a power conversion efficiency of $2.65 \%$ (total area). As the buffer layer composition becomes more Snrich, the $V_{\mathrm{OC}}$ eventually decreases to below that of the control 
Table 1 Photovoltaic characteristics under 1 Sun illumination. After each average value, the standard deviation is indicated

\begin{tabular}{|c|c|c|c|c|c|c|c|c|c|c|}
\hline \multirow[b]{2}{*}{$\begin{array}{l}\text { Buffer } \\
\text { layer }\end{array}$} & \multirow[b]{2}{*}{$\mathrm{Zn} / \mathrm{Sn}$} & \multicolumn{5}{|l|}{ Averaged } & \multicolumn{4}{|l|}{ Best cells } \\
\hline & & $\begin{array}{l}\text { Number } \\
\text { of cells }\end{array}$ & $V_{\mathrm{OC}}(\mathrm{mV})$ & $J_{\mathrm{SC}}\left(\mathrm{mA} \mathrm{cm}^{-2}\right)$ & FF (\%) & Efficiency (\%) & $V_{\mathrm{OC}}(\mathrm{mV})$ & $\begin{array}{l}J_{\mathrm{SC}} \\
\left(\mathrm{mA} \mathrm{cm}{ }^{-2}\right)\end{array}$ & $\mathrm{FF}(\%)$ & Efficiency (\%) \\
\hline $\mathrm{ZnO}$ & - & 18 & $444 \pm 17$ & $7.20 \pm 0.11$ & $59.4 \pm 1.9$ & $1.90 \pm 0.12$ & 462 & 7.26 & 61.8 & 2.07 \\
\hline a-ZTO & $1 / 0.27$ & 17 & $543 \pm 05$ & $7.34 \pm 0.11$ & $62.9 \pm 2.9$ & $2.51 \pm 0.15$ & 553 & 7.37 & 65.0 & 2.65 \\
\hline a-ZTO & $1 / 0.59$ & 17 & $496 \pm 09$ & $7.50 \pm 0.12$ & $61.5 \pm 4.7$ & $2.29 \pm 0.22$ & 503 & 7.56 & 63.4 & 2.41 \\
\hline a-ZTO & $1 / 1.8$ & 18 & $382 \pm 26$ & $7.28 \pm 0.08$ & $54.2 \pm 5.1$ & $1.52 \pm 0.22$ & 406 & 7.25 & 58.2 & 1.72 \\
\hline
\end{tabular}

device. Buffer layers with $\mathrm{Zn}$-to-Sn ratios higher than $1 / 0.27$ do not exhibit further increases in power conversion efficiency.

To determine the mechanism whereby the a-ZTO buffer layer increases the device performance, the band alignment relative to the $\mathrm{Cu}_{2} \mathrm{O}$ layer is characterized by photoelectron spectroscopy following the procedure outlined by Waldrop et al. (Fig. S2$\mathrm{S} 5 \dagger) .{ }^{27}$ An X-ray (Al-K $\alpha$ ) photon source is used to measure the binding energies of $\mathrm{Cu}$ and $\mathrm{Zn}$ core levels with respect to the valence band maximum energy of $\mathrm{Cu}_{2} \mathrm{O}$ and a-ZTO bulk film samples. Two-layer samples that consist of $2.5 \mu \mathrm{m}$ thick $\mathrm{Cu}_{2} \mathrm{O}$ films covered by $\sim 2 \mathrm{~nm}$ thick a-ZTO films are used to measure valence-band energy alignments. The optical (Tauc) gap values are added to estimate conduction-band positions. Fig. 4a shows the estimated band alignments of a-ZTO overlayers on $\mathrm{Cu}_{2} \mathrm{O}$ films. The $\mathrm{Cu}_{2} \mathrm{O}-\mathrm{ZnO}$ interface yields a conduction-band offset $\left(\Delta E_{\mathrm{CB}}\right)$ of $-1.47 \pm 0.2 \mathrm{eV}$ (cliff), comparable to previous reports. ${ }^{28,29}$ All a-ZTO layers are expected to create a conduction band "barrier" greater than $0.3 \mathrm{eV}$, which can prevent electrons in the AZO layer from recombining with holes in the $\mathrm{Cu}_{2} \mathrm{O}$ layer, while allowing photo-generated electrons in the $\mathrm{Cu}_{2} \mathrm{O}$ layer to be collected at the AZO layer. ${ }^{11}$

While the presence of a conduction-band barrier explains the improved performance of the $\mathrm{Zn}$-rich buffer layer, it does not explain the performance reduction observed for increasing Sn concentrations in the buffer layer. We hypothesize that the reduced performance for the Sn-rich buffer layer is due to an increase of subgap states in the buffer layer. Room-temperature capacitance-frequency $(C-f)$ measurements are performed (Fig. 4b); the frequency dependence of diode capacitance is used to measure the densities and energy levels of trap states present in the depletion region. ${ }^{30}$ At high frequencies, the device behaves like an insulator due to dielectric freeze-out and exhibits a geometric capacitance $\left(C_{\mathrm{g}}\right)$. The $C_{\mathrm{g}}$ can be calculated using the relationship $C_{\mathrm{g}}=\varepsilon A / t$, where $\varepsilon$ is the dielectric constant, $A$ is the device area, and $t$ is the thickness of the $\mathrm{Cu}_{2} \mathrm{O}$ layer. At frequencies near $1 \mathrm{MHz}$, capacitances of all devices converge to $4.5-4.7 \mathrm{nF} \mathrm{cm}^{-2}$, close to $C_{\mathrm{g}}$ of the device $(\sim 2.7 \mathrm{nF}$ $\mathrm{cm}^{-2}$ ). At low frequencies, the capacitance of all devices plateaus to a depletion capacitance $\left(C_{\mathrm{d}}\right)$, which is affected by charging and discharging of interface and bulk defect levels present in the depletion region. ${ }^{31}$ The highest efficiency device exhibits the lowest $C_{\mathrm{d}}$, indicative of lower defect densities. On the other hand, the device exhibiting the highest $C_{\mathrm{d}}$ has the lowest $V_{\text {OC }}$ and is Sn-rich (1/1.8). All devices have identical
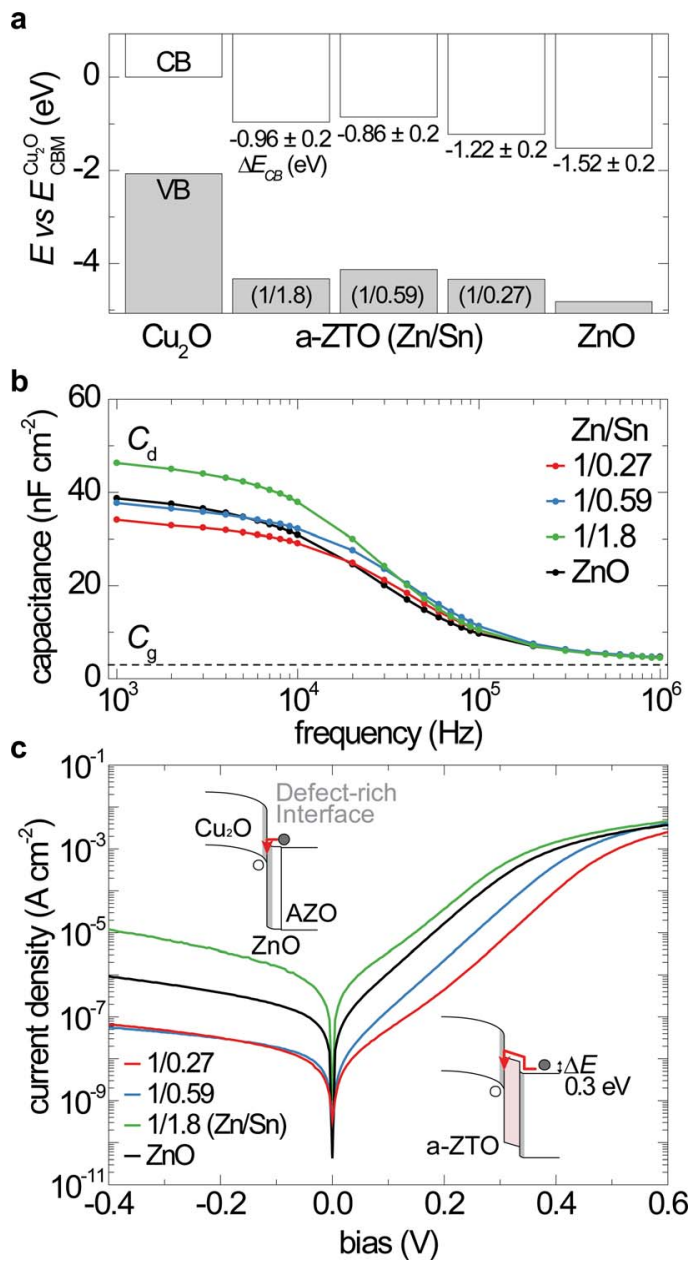

Fig. 4 (a) Relative alignments of conduction-band (CB) and valence-band (VB) energies for a-ZTO and $\mathrm{ZnO}$ overlayers to $\mathrm{Cu}_{2} \mathrm{O}$ thin films investigated in this work, as measured by XPS and optical absorption. (b) Room-temperature capacitance-frequency characteristics of the devices with a-ZTO and ZnO buffer layers measured with $0 \mathrm{~V}(\mathrm{DC})$ and $0.01 \mathrm{~V}(\mathrm{AC})$ bias under dark conditions. (c) Effect of a-ZTO buffer layers on dark current density vs. voltage characteristics of the devices. Inset schematics at the top and bottom show electronic band structures of the devices with $\mathrm{ZnO}$ and a-ZTO $(\mathrm{Zn} / \mathrm{Sn}=1 / 0.27)$ buffer layers, respectively. Grey areas indicate defect-rich interfaces with deep trap states. Red arrow lines represent interface recombination paths for electrons (filled circles) from the AZO layer. The a-ZTO $(Z n / S n=1 / 0.27)$ buffer layer impedes electron movement to the interface where holes (open circles) are provided from the $\mathrm{Cu}_{2} \mathrm{O}$ layer, which reduces $J_{0}$ by nearly a factor of $\sim 40$ compared to the control device (ZnO buffer layer). 
geometry and fabrication processes except for the $5 \mathrm{~nm}$ thick buffer layers. Also, all buffer layer depositions are initiated with a $\mathrm{ZnO}$ sub-cycle to maintain the same atomic interface with the $\mathrm{Cu}_{2} \mathrm{O}$ layer. Thus, the relative change in $C_{\mathrm{d}}$ is likely caused by defects originating from subgap states within the a-ZTO buffer layer. The increased density of subgap states likely promotes recombination, decreasing $V_{\mathrm{OC}}$. Preliminary evidence of increased subgap states in Sn-rich a-ZTO films is provided in Fig. S4. $\dagger$ Although these appear to be "bulk" defect states within the a-ZTO, henceforth we refer to these as "interface states," given the nanometer-scale thickness of the buffer layer.

The $V_{\text {OC }}$ of a heterojunction solar cell is strongly affected by the density and energy levels of interface traps, which increase the dark saturation current by promoting interface recombination. ${ }^{32}$ The $V_{\mathrm{OC}}$ can be estimated as:

$$
V_{\mathrm{OC}} \approx \frac{n k T}{q} \ln \left(\frac{J_{\mathrm{SC}}}{J_{0}}+1\right)
$$

where $n$ is the diode ideality factor, $k$ is the Boltzmann constant, $T$ is temperature, and $q$ is the electron charge. Recombination of carriers through interface traps increases $J_{0}$, thereby reducing the $V_{\text {OC }}$. Dark current density $v s$. voltage $(J-V)$ characteristics of the four devices are plotted on a semi-log scale, as shown in Fig. 4c. The addition of a $5 \mathrm{~nm}$ thick Zn-rich a-ZTO buffer layer reduces $J_{0}$ significantly, resulting in lower dark saturation current densities than the control device (with a $\mathrm{ZnO}$ buffer layer) by a factor of $\sim 40$ under forward bias. Consistent with previous measurements, increasing the Sn content of the buffer layer increases $J_{0}$. When properly tuned, the a-ZTO buffer layer introduces an effective interface-recombination barrier, which impedes the movement of electrons to a defect-rich interface as illustrated in the inset of Fig. 4c. A $5 \mathrm{~nm}$ thick buffer layer is sufficient to block tunnelling while minimizing additional series resistance from the buffer layer.

Additional improvements to $V_{\text {OC }}$ can be gained by enhancing $J_{\mathrm{SC}}$, as given in eqn (1). Due to the low carrier density of electrochemically deposited $\mathrm{Cu}_{2} \mathrm{O}$ thin films $\left(p=10^{13}\right.$ to $10^{14}$ $\mathrm{cm}^{-3}$ ), a fully depleted $\mathrm{Cu}_{2} \mathrm{O}$ layer is expected in our devices. ${ }^{33}$ The photo-generated carrier collection-probability profile in the $\mathrm{Cu}_{2} \mathrm{O}$ layer is modelled to decay exponentially with a minority carrier drift length $\left(L_{\text {drift }}\right)$, due to the high density of lifetimelimiting defects. ${ }^{34}$ We simulate optical absorption in the device by using the finite difference time domain (FDTD) method. The device is modelled as a two-dimensional geometry of AZO (80 $\mathrm{nm}) / \mathrm{a}-\mathrm{ZTO}(5 \mathrm{~nm}) / \mathrm{Cu}_{2} \mathrm{O}(2.5 \mu \mathrm{m}) / \mathrm{Au}(200 \mathrm{~nm})$, with surface texture determined by atomic force microscopy, under incident light with transverse magnetic mode polarization. We used the optical properties of $\mathrm{Cu}_{2} \mathrm{O}$ from ref. 35, since the electrochemically deposited $\mathrm{Cu}_{2} \mathrm{O}$ thin films are too textured to accurately measure the optical path length. Due to the textured top surface morphology and the light-reflecting Au bottom electrode, the device can absorb near band-edge photons effectively. The calculated spatial absorption profile for light with a wavelength of $500 \mathrm{~nm}$ is shown in Fig. 5a. We simulate EQE curves by weighting the generated minority carriers with the spatial collection probability functions with varying $L_{\text {drift }}$ (Fig. S6 $\dagger$ ). Using this method, we generate the EQE curves with calculated a

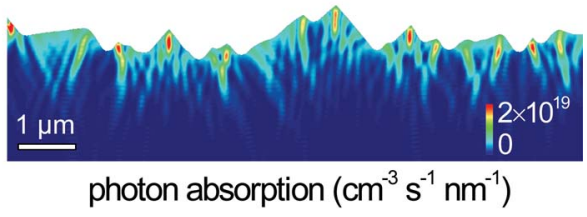

b

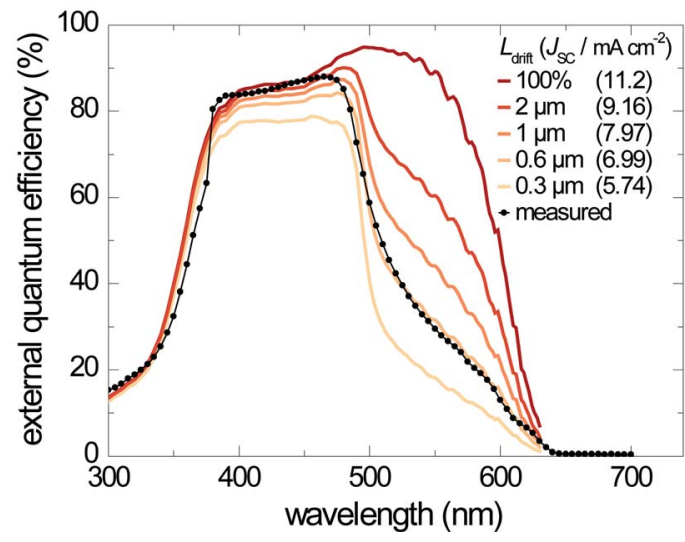

Fig. 5 (a) Simulated optical absorption profile in the $\mathrm{Cu}_{2} \mathrm{O}$ layer for $500 \mathrm{~nm}$ wavelength light with the intensity in the AM1.5G spectrum. (b) Effect of the minority carrier drift length $\left(L_{\text {drift }}\right)$ of $\mathrm{Cu}_{2} \mathrm{O}$ on EQE and $J_{\mathrm{SC}}$. Coloured lines represent calculated EQE from optical simulation with a carrier collection probability profile with increasing $L_{\text {drift }}$ and an ideal $100 \%$ collection case. Numbers in parentheses indicate calculated $J_{\mathrm{Sc}}$. The dotted black line represents measured EQE of the device with an a-ZTO $(\mathrm{Zn} / \mathrm{Sn}=1 / 0.27)$ buffer layer.

$J_{\mathrm{SC}}$ as a function of $L_{\mathrm{drift}}$ and plot measured EQE of the best performing device (1/0.27), as shown in Fig. 5b. The drop-off of EQE for $\lambda<380 \mathrm{~nm}$ is mostly due to absorption by the AZO and a-ZTO layers. The $L_{\text {drift }}$ of the a-ZTO device is estimated to be 0.6 $\mu \mathrm{m}$, by comparing the EQE curves from the FDTD simulation and the actual measurement. The control device shows a similar EQE to the a-ZTO device, re-affirming that the a-ZTO buffer layer affects interface properties only (Fig. S7†). The simulated EQE curves indicate that a $J_{\mathrm{SC}}$ of over $11 \mathrm{~mA} \mathrm{~cm} \mathrm{~cm}^{-2}$ may be possible by increasing the carrier collection efficiency. Improving carrier collection with longer $L_{\text {drift }}$ and light trapping will be an important next step toward achieving devices with higher efficiencies. Further $V_{\text {OC }}$ increases may be achieved by substituting the TCO and buffer layer materials for those with more similar conduction-band energies to $\mathrm{Cu}_{2} \mathrm{O}$.

In summary, we demonstrate an increase of power conversion efficiency of $\mathrm{Cu}_{2} \mathrm{O}$-based all-metal-oxide thin film solar cells by introducing a $5 \mathrm{~nm}$ thick a-ZTO buffer layer between the absorber and TCO. The ALD fabrication process enables a conformal coverage to textured surfaces, as well as tunable electrical and optical properties of the a-ZTO films via the zincto-tin ratio. $J-V$ measurements indicate that the efficiency increase results from the reduction in dark saturation current density. The band-alignment and $C-f$ measurements indicate a reduction of interface recombination, resulting from an electron-blocking barrier by the a-ZTO layer with a low density of trap-states. Due to the highly conformal coverage and precise thickness control by ALD, only a $5 \mathrm{~nm}$ thick buffer layer is necessary to improve the junction quality without sacrificing 
carrier transport and optical transmission. This approach to improve device efficiencies may be extended to other Earthabundant heterojunction photovoltaic cells.

\section{Experimental}

\section{Atomic layer deposition of a-ZTO and AZO thin films}

Amorphous-ZTO, AZO, and undoped $\mathrm{ZnO}$ thin films were synthesized by ALD using a custom-built hot-wall reactor at $120{ }^{\circ} \mathrm{C}$. A cyclic amide of tin (1,3-bis(1,1-dimethylethyl)-4, 5-dimethyl-(4R,5R)-1,3,2-diazastannolidin-2-ylidene)Sn(II) and diethyl zinc (Sigma Aldrich) were used as $\mathrm{Sn}$ and $\mathrm{Zn}$ precursors, respectively. ${ }^{16,19}$ A 50 wt\% hydrogen peroxide $\left(\mathrm{H}_{2} \mathrm{O}_{2}\right)$ solution was used as a common oxidant for a-ZTO growth. A constant flow of $\mathrm{N}_{2}$ carrier gas was employed during the ALD of ZTO. ${ }^{16}$ The tin precursor and diethyl zinc were held at 40 and $25^{\circ} \mathrm{C}$ and their equilibrium vapour pressures were estimated to be 0.42 and 6.8 Torr, respectively. The exposures of the tin precursor and diethyl zinc were estimated to be around 0.33 and 0.032 Torr $\mathrm{s}$ in each cycle, respectively. The exposures of $\mathrm{H}_{2} \mathrm{O}_{2}$ and $\mathrm{H}_{2} \mathrm{O}$ were about 0.027 and 4.4 Torr s, respectively. To deposit aZTO films, an ALD super-cycle scheme with $\mathrm{ZnO}$ and $\mathrm{SnO}_{2}$ subcycles was employed. Three different $\mathrm{ZnO} / \mathrm{SnO}_{2}$ sub-cycle ratios of $3 / 1,1 / 1,1 / 3$ were used to find the optimized composition as a buffer layer in the $\mathrm{Cu}_{2} \mathrm{O}-\mathrm{ZnO}$ heterojunction. Their nominal growths per one super-cycle were 3.1,1.6 and 4.1 $\AA$, respectively, measured by X-ray reflectivity. AZO thin films were also synthesized at $120{ }^{\circ} \mathrm{C}$ by ALD using diethyl zinc, trimethylaluminum and de-ionized water as $\mathrm{Zn}$, $\mathrm{Al}$ precursors and oxidant, respectively. An optimized $\mathrm{Al}_{2} \mathrm{O}_{3}$ doping ratio to $\mathrm{ZnO}$ ( 1 cycle of $\mathrm{Al}_{2} \mathrm{O}_{3}$ after every 19 cycles of $\mathrm{ZnO}$ ) was selected to obtain the lowest resistivity $\left(5.9 \times 10^{-3} \Omega \mathrm{cm}\right)$ of the films. The nominal film growth per one super-cycle, which consists of 19-ZnO and 1- $\mathrm{Al}_{2} \mathrm{O}_{3}$ sub-cycles, was $32 \AA$. Undoped $\mathrm{ZnO}$ as a control buffer layer was grown at $120{ }^{\circ} \mathrm{C}$ without $\mathrm{Al}$ doping with de-ionized water as an oxidant.

\section{Thin film solar cell fabrication}

An Au bottom electrode ( $200 \mathrm{~nm}$ thick, $3.2 \mathrm{~cm}^{2}$ area) with a $5 \mathrm{~nm}$ thick Ti adhesion layer was deposited on a $1 \times 1 \mathrm{inch}^{2}$ fused silica by e-beam evaporation. A $2.5 \mu \mathrm{m}$ thick $\mathrm{Cu}_{2} \mathrm{O}$ film was deposited on the Au film at $40{ }^{\circ} \mathrm{C}$ by the galvanostatic electrochemical method ${ }^{36}$ A lactate-stabilized copper sulfate aqueous solution was prepared with $3 \mathrm{M}$ lactic acid (Sigma Aldrich), $0.2 \mathrm{M}$ cupric sulfate pentahydrate $\left(\mathrm{CuO}_{4} \mathrm{~S} \cdot 5 \mathrm{H}_{2} \mathrm{O}\right.$, Sigma Aldrich) and de-ionized water (18.3 $\mathrm{M} \Omega \mathrm{cm}$, Ricca Chemical) was prepared and $2 \mathrm{M}$ sodium hydroxide $(\mathrm{NaOH}$, Sigma Aldrich) aqueous solution was added to adjust the $\mathrm{pH}$ of the solution to 12.5. All reagent-grade chemicals were used and the solution was filtered and stirred thoroughly. A constant current density of $0.23 \mathrm{~mA} \mathrm{~cm}^{-2}$ was applied using a Keithley 2400 sourcemeter with a Pt counter electrode to grow the $\mathrm{Cu}_{2} \mathrm{O}$ films. $\mathrm{The} \mathrm{Cu}_{2} \mathrm{O}$ film surfaces were rinsed with de-ionized water. AmorphousZTO and polycrystalline AZO layers were deposited by ALD, followed by Al electrode deposition. The $\mathrm{Al}$ electrodes $(300 \mathrm{~nm}$ thick) were deposited by e-beam evaporation with a grid spacing of $1 \mathrm{~mm}$ defined by a shadow mask. A $3 \times 5 \mathrm{~mm}^{2}$ cell area was defined by photolithography and wet etching with a nitric acid solution.

\section{Characterization}

The microstructures of the films were characterized by JEM-2100 HR-TEM (JEOL) and GAXRD using a PANalytical X'Pert Pro diffractometer with $\mathrm{Cu}-\mathrm{K} \alpha$ radiation $\left(\omega=0.9^{\circ}\right)$. Surface morphologies of the grown films were analysed using an Ultra 55 FESEM (Zeiss). Optical properties of the films were measured using a U-4100 UV-VIS spectrophotometer (Hitachi) and a VASE spectroscopic ellipsometer (J. A. Woollam Co., Inc.). Electrical properties of the films were characterized by Hall effect measurements using the van der Pauw configuration and a magnetic field of $0.75 \mathrm{~T}$. XPS and UPS measurements were performed using a PHI VersaProbe II (Physical Electronics) and an AXIS Nova (Kratos analytical, KBSI Jeonju, Korea). Samples were etched by an Ar ion beam to remove carbon contaminants on the surface. The current-voltage and the capacitance-frequency characteristics of the device were measured by using Agilent 4156C and Keithley 4200 semiconductor characterization systems. The standard 1 Sun illumination was generated by a Newport Oriel 91194 solar simulator with a 1300 W Xe-lamp with a AM1.5G filter and a Newport Oriel 68951 flux controller calibrated by an NREL-certified Si reference cell equipped with a BG39 window. No intentional light-soaking was applied to the devices. EQE of the device was measured using a QEX7 (PV Measurements, Inc.) calibrated by a NIST-certified Si photodiode. The top surface morphology of the device was measured by atomic force microscopy using a MFP-3D SA (Asylum Research).

\section{Acknowledgements}

The authors thank Dr I. Kozinsky (Bosch), B. Son (KBSI), and K. Broderick (MIT) for helpful discussions and experimental support. F. Frankel (MIT) is thanked for helpful advice on graphics. This work was supported by the Chesonis Family Foundation, Bosch through the MIT Energy Initiative, NSF award CBET-1032955, and NSF CAREER award ECCS-1150878. This work made use of the MTL and CMSE at MIT and the CNS at Harvard University supported by NSF awards DMR-0819762 (CMSE) and ECS-0335765 (CNS). A Clean Energy Scholarship from NRF Singapore (S.C.S.) and an NSF Graduate Research Fellowship (R.E.B.) are acknowledged.

\section{Notes and references}

1 C. Wadia, A. P. Alivisatos and D. M. Kammen, Environ. Sci. Technol., 2009, 43, 2072-2077.

2 K. P. Musselman, A. Wisnet, D. C. Iza, H. C. Hesse, C. Scheu, J. L. MacManus-Driscoll and L. Schmidt-Mende, Adv. Mater., 2010, 22, E254-E258.

3 S. Rühle, A. Y. Anderson, H.-N. Barad, B. Kupfer, Y. Bouhadana, E. Rosh-Hodesh and A. Zaban, J. Phys. Chem. Lett., 2012, 3, 3755-3764.

4 B. K. Meyer, A. Polity, D. Reppin, M. Becker, P. Hering, P. J. Klar, T. Sander, C. Reindl, J. Benz, M. Eickhoff, 
C. Heiliger, M. Heinemann, J. Bläsing, A. Krost, S. Shokovets, C. Müller and C. Ronning, Phys. Status Solidi B, 2012, 249, 1487-1509.

5 C. Xiang, G. M. Kimball, R. L. Grimm, B. S. Brunschwig, H. A. Atwater and N. S. Lewis, Energy Environ. Sci., 2011, 4, 1311-1318.

6 Y. Nishi, T. Miyata and T. Minami, Thin Solid Films, 2013, 528, 72-76.

7 M. Izaki, T. Shinagawa, K. T. Mizuno, Y. Ida, M. Inaba and A. Tasaka, J. Phys. D: Appl. Phys., 2007, 40, 3326-3329.

8 S. Jeong, S. H. Song, K. Nagaich, S. A. Campbell and E. S. Aydil, Thin Solid Films, 2011, 519, 6613-6619.

9 K. P. Musselman, A. Marin, A. Wisnet, C. Scheu, J. L. MacManus-Driscoll and L. Schmidt-Mende, Adv. Funct. Mater., 2011, 21, 573-582.

10 M. Taguchi, A. Terakawa, E. Maruyama and M. Tanaka, Prog. Photovoltaics, 2005, 13, 481-488.

11 T. Minemoto, T. Matsui, H. Takakura, Y. Hamakawa, T. Negami, Y. Hashimoto, T. Uenoyama and M. Kitagawa, Sol. Energy Mater. Sol. Cells, 2001, 67, 83-88.

12 J. Robertson, Phys. Status Solidi B, 2008, 245, 1026-1032.

13 A. Walsh, J. L. F. D. Silva and S.-H. Wei, J. Phys.: Condens. Matter, 2011, 23, 334210.

14 E. Fortunato, P. Barquinha and R. Martins, Adv. Mater., 2012, 24, 2945-2986.

15 K. Nomura, H. Ohta, A. Takagi, T. Kamiya, M. Hirano and H. Hosono, Nature, 2004, 432, 488-492.

16 J. Heo, S. B. Kim and R. G. Gordon, Appl. Phys. Lett., 2012, 101, 113507.

17 J. Lindahl, J. T. Wätjen, A. Hultqvist, T. Ericson, M. Edoff and T. Törndahl, Prog. Photovoltaics, 2012, DOI: 10.1002/pip. 2239.

18 Z. Zhang, W. Hu, Y. Deng, C. Zhong, H. Wang, Y. Wu and L. Liu, Mater. Res. Bull., 2012, 47, 2561-2565.

19 J. Heo, A. S. Hock and R. G. Gordon, Chem. Mater., 2010, 22, 4964-4973.

20 H. Q. Chiang, J. F. Wager, R. L. Hoffman, J. Jeong and D. A. Keszler, Appl. Phys. Lett., 2005, 86, 013503.
21 J.-Y. Kwon, D.-J. Lee and K.-B. Kim, Electron. Mater. Lett., 2011, 7, 1-11.

22 T. Moriga, Y. Hayashi, K. Kondo, Y. Nishimura, K.-i. Murai, I. Nakabayashi, H. Fukumoto and K. Tominaga, J. Vac. Sci. Technol., A, 2004, 22, 1705-1710.

23 Y.-H. Kim, J.-S. Heo, T.-H. Kim, S. Park, M.-H. Yoon, J. Kim, M. S. Oh, G.-R. Yi, Y.-Y. Noh and S. K. Park, Nature, 2012, 489, 128-132.

24 S. Narushima, H. Mizoguchi, K. Shimizu, K. Ueda, H. Ohta, M. Hirano, T. Kamiya and H. Hosono, Adv. Mater., 2003, 15, 1409-1413.

25 K. Satoh, Y. Kakehi, A. Okamoto, S. Murakami, F. Uratani and T. Yotsuya, Jpn. J. Appl. Phys., 2005, 44, L34-L37.

26 J. S. Rajachidambaram, S. Sanghavi, P. Nachimuthu, V. Shutthanandan, T. Varga, B. Flynn, S. Thevuthasan and G. S. Herman, J. Mater. Res., 2012, 27, 2309-2317.

27 J. R. Waldrop, R. W. Grant, S. P. Kowalczyk and E. A. Kraut, J. Vac. Sci. Technol., A, 1985, 3, 835-841.

28 Z. Q. Duan, A. Du Pasquier, Y. C. Lu, Y. Xu and E. Garfunkel, Sol. Energy Mater. Sol. Cells, 2012, 96, 292-297.

29 L. M. Wong, S. Y. Chiam, J. Q. Huang, S. J. Wang, J. S. Pan and W. K. Chim, J. Appl. Phys., 2010, 108, 033702.

30 T. Walter, R. Herberholz, C. Muller and H. W. Schock, J. Appl. Phys., 1996, 80, 4411-4420.

31 T. Eisenbarth, T. Unold, R. Caballero, C. A. Kaufmann and H.-W. Schock, J. Appl. Phys., 2010, 107, 034509-034512.

32 M. Saad and A. Kassis, Sol. Energy Mater. Sol. Cells, 2003, 79, 507-517.

33 K. P. Musselman, A. Marin, L. Schmidt-Mende and J. L. MacManus-Driscoll, Adv. Funct. Mater., 2012, 22, 22022208.

34 K. P. Musselman, Y. Ievskaya and J. L. MacManus-Driscoll, Appl. Phys. Lett., 2012, 101, 253503.

35 C. Malerba, F. Biccari, C. L. A. Ricardo, M. D'Incau, P. Scardi and A. Mittiga, Sol. Energy Mater. Sol. Cells, 2011, 95, 28482854.

36 P. E. de Jongh, D. Vanmaekelbergh and J. J. Kelly, Chem. Mater., 1999, 11, 3512-3517. 